\title{
The pandemic paradox: Delay in the treatment of a woman with endometrial cancer during COVID-19 outbreak
}

\author{
Konstantinos Zacharis, Polyxeni Vanakara, Alexandros Daponte
}

Department of Obstetrics and Gynecology, Faculty of Medicine, School of Health Sciences, University of Thessaly, Larissa, Greece

\section{Corresponding Author}

Zacharis Konstantinos, Department of Obstetrics and Gynecology, University Hospital of Larissa, University of Thessaly, Viopolis, 41500, Larissa, Greece. Tel.: +30 2413502795, e-mail: zaxarisk@yahoo.com

Dear Editor,

The novel coronavirus (COVID-19) outbreak has been declared a global pandemic. The containment measures implemented in recent weeks to tackle it have led in drastic alterations in people's daily life. These changes were essential to reduce virus transmission and protect health systems ${ }^{1}$.

However, lockdown and strict measures, including home quarantine and social distancing, have delayed the diagnosis or postponed the therapeutic management of patients with neoplasia, such as surgery, systemic chemotherapy and radiation. On the other hand, COVID-19 exposure may have adverse outcomes in patients with cancer because of their systemic immunosuppression caused by the malignancy or anticancer treatment methods ${ }^{2}$.

We present a bizarre case of delay in the treatment of a woman with endometrial cancer. A 79-yearold Caucasian woman presented to our outpatient department in May 2020 with a diagnosis of endometrial cancer since February 2020. Endometrial cancer was diagnosed in a secondary institution by dilation and curettage due to postmenopausal vaginal bleeding.

Histopathology revealed a malignant mixed Müllerian tumor. The epithelial component was compatible with a grade 3 endometrioid adenocarcinoma. Pretreatment clinical staging tests including chest $\mathrm{X}$-ray, ultrasound and magnetic resonance imaging scan of the pelvis were performed. Significant finding was endometrial cancer with invasion of more than half of myometrium, clinical stage IB. Thus, patient was referred to a tertiary hospital.

Meanwhile the lockdown was implemented in Greece, therefore the patient along with her family members decided to postpone the clinic appointment to our department, in order to reduce the risk of viral transmission.

In May 2020, while COVID-19 lockdown measures were gradually eased the patient presented to our outpatient department. On admission, the patient described that during the home quarantine she experienced multiple episodes of vaginal bleeding and she used tranexamic acid orally without a medical prescription. From past medical history, she reported arterial hypertension, diabetes mellitus 
and aortic valve stenosis. Her BMI was $45.4 \mathrm{~kg} / \mathrm{m} 2$. Bimanual pelvic examination revealed heavy vaginal bleeding and transvaginal ultrasonography showed an enlarged uterus with an endometrial thickness of $24 \mathrm{~mm}$.

Due to the wait interval of four months and the clinical findings, we also performed a restaging imaging. A contrast enhanced computed tomography scan of abdomen showed an invasion of the left parametrium as well as multiple enlarged pelvic and paraaortic lymph nodes, implying a progression from Stage IB to Stage IIIC.

The multidisciplinary team discussed our case and the patient was disqualified from surgery due to the severity of the disease (FIGO -Stage IIIC) and patient's advanced age and poor general condition; therefore, radiotherapy was recommended. The patient received external pelvic radiotherapy with 16 applications of $250 \mathrm{cGy} /$ day (total dose of 4,000 cGy).

It is popularly assumed, that delay in the delivery of treatment for uterine cancer have a negative impact on overall survival ${ }^{3}$, whilst a study from Israel showed that a delay in treatment of four months do not compromise survival of patients with endometrial cancer ${ }^{4}$.

Although total hysterectomy with bilateral salpingo-oophorectomy is considered the mainstay treatment for endometrial cancer, our case demonstrates that a delay in the initial evaluation and postponement of the therapeutic management, due to COVID-19 related transmission fear and anxiety in the pandemic process, consequently lead to cancer development, modification of treatment plan and deterioration of patient's prognosis.

Hence, in patients with advanced oncologic disease, the benefit of intervention must be balanced with the risk for potential COVID-19 exposure in health care facilities. Postponement in cancer treatment should be individualized because delay could lead to tumor progression and may affect the ultimate prognosis of patients ${ }^{5}$.

\section{Conflict of interest}

The authors declare no conflict of interest.

\section{References}

1. Davies NG, Kucharski AJ, Eggo RM, Gimma A, Edmunds WJ; Centre for the Mathematical Modelling of Infectious Diseases COVID-19 working group. Effects of non-pharmaceutical interventions on COVID-19 cases, deaths, and demand for hospital services in the UK: a modelling study. Lancet Public Health. 2020;5:(7):e375-e385. doi:10.1016/ S2468-2667(20)30133-X.

2. Kutikov A, Weinberg DS, Edelman MJ, Horwitz EM, Uzzo RG, Fisher RI. A War on Two Fronts: Cancer Care in the Time of COVID-19. Ann Intern Med. 2020;172(11):756-758. doi:10.7326/M20-1133

3. Pergialiotis V, Haidopoulos D, Tzortzis AS, Antonopoulos I, Thomakos N, Rodolakis A. The impact of waiting intervals on survival outcomes of patients with endometrial cancer: A systematic review of the literature. Eur J Obstet Gynecol Reprod Biol. 2020;246:1-6. doi:10.1016/j.ejogrb.2020.01.004

4. Menczer J, Krissi H, Chetrit A, et al. The effect of diagnosis and treatment delay on prognostic factors and survival in endometrial carcinoma [published correction appears in Am J Obstet Gynecol 1996 Dec;175(6):1678. Gaylor J [corrected to Gaynor J]]. Am J Obstet Gynecol. 1995;173[3 Pt 1):774-778. doi:10.1016/0002-9378(95)90340-2

5. Wang H, Zhang L. Risk of COVID-19 for patients with cancer. Lancet Oncol. 2020;21(4):e181. doi:10.1016/S1470-2045(20)30149-2

Received 11-05-21

Revised 14-05-21

Accepted 18-05-21 